\title{
Effect of an exclusion range of jaw movement data from the intercuspal position on the estimation of the kinematic axis point
}

\section{Authors:}

Shuji Shigemoto, DDS, $\mathrm{PhD}^{1}$

${ }^{1}$ Department of Fixed Prosthodontics, Institute of Health Biosciences, The University of

Tokushima Graduate School, Tokushima, Japan

Nobuyuki Bando, DDS, $\mathrm{PhD}^{1}$

${ }^{1}$ Department of Fixed Prosthodontics, Institute of Health Biosciences, The University of

Tokushima Graduate School, Tokushima, Japan

Keisuke Nishigawa, DDS, $\mathrm{PhD}^{2}$

${ }^{2}$ General Dentistry, Tokushima University Hospital, Tokushima, Japan

Yoshitaka Suzuki, DDS, $\mathrm{PhD}^{2}$

${ }^{2}$ General Dentistry, Tokushima University Hospital, Tokushima, Japan

Toyoko Tajima, DDS, $\mathrm{PhD}^{2}$

${ }^{2}$ General Dentistry, Tokushima University Hospital, Tokushima, Japan

Kazuo Okura, DDS, $\mathrm{PhD}^{1}$

${ }^{1}$ Department of Fixed Prosthodontics, Institute of Health Biosciences, The University of

Tokushima Graduate School, Tokushima, Japan

*Yoshizo Matsuka, DDS, $\mathrm{PhD}^{1}$

${ }^{1}$ Department of Fixed Prosthodontics, Institute of Health Biosciences, The University of

Tokushima Graduate School, Tokushima, Japan

*Corresponding author

Prof. Yoshizo Matsuka

Department of Fixed Prosthodontics, Institute of Health Biosciences, The University of

Tokushima Graduate School, Tokushima, Japan

3-18-15, Kuramoto-cho, Tokushima, Tokushima 770-8504, Japan

TEL: +8188633 7350, FAX: +81886337391

e-mail: matsuka@tokushima-u.ac.jp 
1 Abstruct

2 In patients who have lost natural occlusal contacts, the centric relation is usually estimated based on

3 several anatomical factors such as the temporomandibular joint and masticatory muscles except

4 dental arch. The clinical procedure to record the centric relation often depends on the clinician's

5 expertise and technique; an objective method to determine proper occlusal position is desirable. The

6 kinematic axis point (KAP) is kinematically estimated from sagittal movements and is an ideal

7 posterior reference point that is used in dental articulators for reproducing jaw movement. Occlusal

8 registration using the KAP may serve as a definite objective technique. The aim of this study is to

9 investigate the effect of the exclusion range of sagittal jaw movement data from the intercuspal

10 position (ICP) on the estimation of the KAP. The complete and incomplete sagittal border movement

11 data of dentate subjects were used to estimate the KAPs. The locations of the estimated KAPs were

compared. The results indicate that the incomplete sagittal border jaw movement data set does not 
Introduction

The occlusal position at maximum intercuspation is determined by several anatomical factors

such as the dental arch, temporomandibular joint, and masticatory muscles [1]. In patients who have

lost natural occlusal contacts because of missing teeth, the centric relation is usually estimated based

on factors except dental arch [2]. As the clinical procedure to record centric relation, i.e., occlusal

registration often depends on the clinician's expertise and technique, an objective method to

determine proper occlusal position is desirable. Kohno studied the jaw movement of subjects with a

natural intercuspal position (ICP) and reported that the sagittal jaw movement pathway exhibits convergence at the mandibular condyle head area. He named this point the kinematic axis point (KAP) and the line that connects the KAPs on both sides the kinematic axis (KA) [3-5]. Mathematically, the KA separates the translational and rotational components of sagittal jaw movement and enables the simple expression of jaw movement $[3,4,6,7]$. Because of this kinematic property, the KAP is an ideal posterior reference point that is used in dental articulators to reproduce jaw movement for the fabrication of prosthetic appliances. Since the KAP can be kinematically estimated by computing jaw movement data, occlusal registration using the KAP as the posterior reference point may serve as a definite objective technique with a theoretical background. To confirm the usability of this technique, an accurate determination of the KAP is indispensable. In this 

of the KAP.

Forty-five adult volunteers ( 23 men and 22 women; mean age, $26.6 \pm 6.7$ years) selected from among the faculties and students of Tokushima University Dental School participated in this research. All subjects underwent an examination with questionnaires and palpation of head and neck region according to the questionnaires and clinical protocol of the Temporpmandibular Disorder Clinic, Tokushima University Hospital. All subjects had normal dentition and stable occlusal contacts at the ICP. None of the subjects had missing teeth except for the third molar and had received orthodontic treatment within the past year. No signs or symptoms of temporomandibular disorders including pain and dysfunction in the temporomandibular joint and/or the masticatory muscles were present in any of the subjects. This research was approved by the Research Ethics Committee of Tokushima University Hospital (No. 575). The informed consent was obtained from all participants prior to data collection. 
A custom-made six degree-of-freedom jaw tracking device using a magnetic sensor system (Fig.

1) $[8,9]$ was used to record the jaw movement. This device uses a pair of miniature triaxial coils connected to the maxillary and mandibular dentitions. Each sensor unit is a $21 \mathrm{~mm}$ cube weighing $7.0 \mathrm{~g}$. The calibration study revealed the measurement errors of this device were better than $48 \mu \mathrm{m}$ for translation and $0.035^{\circ}$ for rotation $[8,9]$. An acrylic splint attached to the labial surface of the dentition was used to mount the sensor unit. The primary sensor unit (Fig. 1a) generates triaxial magnetic fields and is placed in the anterior area of the nose. The secondary sensor unit (Fig. 1b) is placed in the left lateral area of the buccal region and detects magnetic field changes. These sensor units were initially placed $100 \mathrm{~mm}$ apart from each other, i.e., the ICP of the jaw movement recording (Fig. 1). Each subject was asked to sit upright in a dental chair without the head supported and to start jaw movements from the ICP. Each participant performed three sagittal border jaw movements that were measured separately with a sampling rate of $100 \mathrm{~Hz}$. To analyze and display jaw movement data, we employed the upper jaw coordinate system $\left(\mathrm{O}_{\mathrm{u}}-\mathrm{x}_{\mathrm{u}} \mathrm{y}_{\mathrm{u}} \mathrm{z}_{\mathrm{u}}\right)[10]$ as the reference coordinate system, as shown in Fig. 2. The origin of this system is located at the centroid of the triangle defined by an incisal point (IN, central point of the medial edge of the maxillary incisor) and the left and 

$\mathrm{y}_{\mathrm{u}^{-}}$, and $\mathrm{z}_{\mathrm{u}}$-axes point in the subject's anterior, left, and superior directions, respectively. The positive rotation directions are defined by the right-hand rule.

\section{Estimation algorithm for the KAP}

As shown in Fig. 2, the intercondylar axis (ICA) is initially determined based on the Bonwill

triangle with a side length of $110 \mathrm{~mm}$ and a Balkwill angle of $20^{\circ}$, and 13 distinct points $\mathrm{P}_{\mathrm{m}}(\mathrm{m}$ :

$1 \ldots 13$ ) are arranged at 10-mm intervals on the ICA. $\mathrm{P}_{2}$ and $\mathrm{P}_{12}$ are included in the Bonwill triangle as the right and left condylar points, respectively. A two-dimensional (2D) projection of the sagittal

border jaw movement pathway of $\mathrm{P}_{\mathrm{m}}$ in a particular sagittal plane $\mathrm{V}_{\mathrm{m}}$ that passes through $\mathrm{P}_{\mathrm{m}}$ is used to determine the KAP. $V_{m}$ is perpendicular to the ICA (Fig. 2). The least squares circle in $V_{m}$ that fits the set of the 2D projection sagittal border jaw movement pathway is found. The circumference of the circle is divided into $\mathrm{n}$ arcs of $0.2 \mathrm{~mm}$ using dotted rays that start at its center, and then the maximum radial thicknesses $r t h_{i}(\mathrm{i}=1 \ldots \mathrm{n})$ of the jaw movement data between two adjacent dotted rays is calculated (Fig. 3). The sum $\sum_{i=1}^{i=n} r t h_{i}^{2}$ of the squares of the radial thickness $\left(\underline{r t h} \underline{i}_{i}\right)$ is employed as a geometric parameter to determine the KAP. The $\mathrm{KAP}$ in $\mathrm{V}_{\mathrm{m}}\left(\mathrm{KAP}_{\mathrm{m}}\right)$ is obtained as a point minimizing the value of this geometric parameter. As shown in Fig. 4, the searching procedure is started from $5 \times 5$ grid points spaced $10 \mathrm{~mm}$ apart, the center of which is set to $\mathrm{P}_{\mathrm{m}}$. The point $\left(\mathrm{MP}_{\mathrm{m}}\right)$ minimizing the value of the geometric parameter is chosen from among these 25 points, and then the 
92 grid interval, and the center is set to $\mathrm{MP}_{\mathrm{m}}$. This searching procedure is repeated nine times (grid interval $=0.039 \mathrm{~mm}$ ), and finally, the last $\mathrm{MP}_{\mathrm{m}}$ value is selected as the $\mathrm{KAP}_{\mathrm{m}}$. Additionally, its

94 quadratic mean radial thickness $\sqrt{\frac{1}{n} \sum_{i=1}^{i=n} r t h_{i}^{2}}$ is calculated. Using 3D linear regression, we 95 determined KA such that the sum $\sum_{m=1}^{m=13} d_{m}^{2}$ of the squares of the perpendicular distances $\left(d_{m}\right)$ between 96 the $\mathrm{KAP}_{\mathrm{m}}$ and the line is minimized, and its quadratic mean perpendicular distance $\sqrt{\frac{1}{13} \sum_{m=1}^{m=13} d_{m}^{2}}$ is calculated. We decided $\mathrm{KAP}_{2}$ and $\mathrm{KAP}_{12}$ were the right and left condylar points, respectively. We used all of the $\mathrm{KAP}_{\mathrm{m}}$ for the following analysis. This searching procedure was performed automatically using a custom application that was created in Microsoft Visual C++ 2008. (exc_KAP) estimate the KAPs. The complete data set, including all of the recorded data, ranges from the ICP to the maximum protrusion position (Pr) and then to the maximum opening position (MO) through the anterior border movement pathway, and then from the MO to the ICP through the posterior border 
108 points in the exclusion range of a $15 \mathrm{~mm}$ distance from the ICP. The data exclusion range was

109 changed each $1 \mathrm{~mm}$ (Fig. 5B).

110 Using the estimation algorism mentioned above, the reference $\mathrm{KAP}_{\mathrm{m}}\left(\mathrm{ref}_{-} \mathrm{KAP}_{\mathrm{m}}\right)$ and $\mathrm{KA}$

111 (ref_KA) were estimated from three complete sagittal border movement data sets. In the same

112 manner, the exclusion $\mathrm{KAP}_{\mathrm{m}}\left(\mathrm{exc}_{-} \mathrm{KAP}_{\mathrm{m}}\right)$ and $\mathrm{KA}\left(\mathrm{exc}_{-} \mathrm{KA}\right)$ were estimated from three excluded

113 sagittal border movement data sets for each exclusion range. The mean positional deviations

114 (antero-posterior $(\mathrm{dx})$, supero-inferior $(\mathrm{dz})$, and Euclidean distance $(\mathrm{d}))$ between the exc_KAP $\mathrm{m}_{\mathrm{m}}$ and

115 the ref_KAP ${ }_{\mathrm{m}}$ of 45 subjects were calculated for each exclusion range (Fig. 6).

117 5. Statistical analysis

118 Paired $t$-test was used to compare the values of the radial thicknesses of the pathways of the

119 ref_KAP ${ }_{2}$ (right condylar point) and those of the ref_KAP 12 (left condylar point). To evaluate effect

120 of the data exclusion range on the validity of the KAP estimation, a segmented linear regression

121 analysis with two segments was used to determine the relationship between the 2D position

122 deviation of the exc_KAP from the ref_KAP and the range of the exclusion area and two regression

123 slopes were compared by means of a one-way analysis of covariance (ANCOVA) to analyze the

124 effect of the range of data exclusion on the position of exc_KAP. The level of significance was P < 
127 Results

1. Estimation algorithm for the KAP

129

The pathways of axis points $\left(\mathrm{P}_{2}, \mathrm{P}_{7}\right.$, and $\mathrm{P}_{12}$ for the ICA and $\mathrm{KAP}_{2}, \mathrm{KAP}_{7}$, and $\mathrm{KAP}_{12}$ for $\left.\mathrm{KA}\right)$

130

are illustrated in Fig. 7. As a result of analyzing the complete sagittal border movement data of 45

131 subjects, the estimated ref_KAP $\mathrm{K}_{\mathrm{m}}(\mathrm{m}: 1 \ldots 13)$ pathway showed a narrow, belt-shaped range and the

average of the quadratic mean radial thickness of the right $\left(\right.$ ref_KAP 2 ) and left (ref_KAP $\left.{ }_{12}\right)$ condylar

133 points pathway (mean $\pm \mathrm{SD} ; \mathrm{n}=45$ ) were $0.96 \pm 0.31 \mathrm{~mm}$ and $0.89 \pm 0.40 \mathrm{~mm}$, respectively. There

were no significant differences between the radial thickness of the right and left condylar points

135 (Paired $t$-test, $\mathrm{p}=0.20$ ). Furthermore, the ref_KAP $\mathrm{m}$ was located mostly on the ref_KA, and the

136 average of the quadratic mean perpendicular distance from the ref_KAP $\mathrm{m}_{\mathrm{m}}$ to the ref_KA (mean $\pm \mathrm{SD}$; $\mathrm{n}=45$ ) was $0.36 \pm 0.24 \mathrm{~mm}$ (Fig. 7).

2. Estimation of exc_KAP from the excluded sagittal border movement data

Fig. 8 shows the relationship between the data exclusion range and the mean positional

141 deviation between the exc_KAP ${ }_{m}$ and the ref_KAP $m(m: 1 \ldots 13)$. The antero-posterior $(\mathrm{dx})$ and

142 supero-inferior $(\mathrm{dz})$ mean positional deviations of the exc_KAP $\mathrm{m}_{\mathrm{m}}$ from the ref_KAP $\mathrm{K}_{\mathrm{m}}$ (mean $\pm \mathrm{SD}$; 
and $-0.26 \pm 0.30 \mathrm{~mm}$ with a 7-mm data exclusion range, and $1.18 \pm 0.26 \mathrm{~mm}$ and $-1.64 \pm 0.23 \mathrm{~mm}$

145 with a 13-mm data exclusion range, respectively. In addition, the mean Euclidean distances (d)

146 between ref_KAP ${ }_{m}$ and $\exp _{-} \mathrm{KAP}_{\mathrm{m}}($ mean $\pm \mathrm{SD} ; \mathrm{n}=13)$ were $0.12 \pm 0.02 \mathrm{~mm}$ with a 1-mm data

147 exclusion range, $0.47 \pm 0.13 \mathrm{~mm}$ with a 7 - $\mathrm{mm}$ data exclusion range, and $2.12 \pm 0.25 \mathrm{~mm}$ with a

148 13-mm data exclusion range. The exc_KAP position gradually deviated in the antero-inferior

149 direction from the ref_KAP. There were two linear relationships in the data until the exclusion range

150 of $13 \mathrm{~mm}$ and a segmented linear regression analysis detected the breakpoints (BP) of $8.51 \mathrm{~mm}$ for

$151 \mathrm{dx}, 7.64 \mathrm{~mm}$ for $\mathrm{dz}$, and $8.36 \mathrm{~mm}$ for d, respectively. Slopes below the BP were $0.0290 \mathrm{for} \mathrm{dx}$,

$152-0.0373$ for $\mathrm{dz}$, and 0.0649 for $\mathrm{d}$, respectively and those above the BP were 0.2121 for $\mathrm{dx},-0.2571$

153 for $\mathrm{dz}$, and 0.3355 for $\mathrm{d}$, respectively. To test for differences in slopes among the regression lines

154 below and above the BP, we conducted a one-way ANCOVA with exclusion range as a covariate and the exc_KAP position deviation as the dependent variable. The slopes below and above the BP were significantly different $(\mathrm{p}<0.05)$ in all position deviations $(\mathrm{dx}, \mathrm{dz}$, and $\mathrm{d})$. In other word, there was a significant change in the position deviation rates below and above the BP. The effect of the exclusion range on the exc_KAP position deviation below the BP was smaller than that above the BP.

Discussion 
162 [11]. The hinge axis is widely used as the condylar axis for reproduce of jaw movements in a

163 mechanical articulator because of its easy identification and reproducible [6,11,12]. However, the

164 movement paths of the hinge axis depends upon the rotatory component of mandibular movements

165 and are representative of those of the subject's condyle during only the terminal hinge movements

166 [6,11]. While, KA separates the translational and rotational components of the various sagittal

167 movements $[3,4,6,7]$, it can be an ideal condylar reference axis. If it would be possible that KA

168 coincides with the ICA of the mechanical articulator, the various types of jaw movements in the

169 sagittal plane can be reproduced in the articulator and thus the design of dental prosthesis is

170 improved. Beneficially, KA is mathematically estimated from jaw movement data without the

171 necessity of the use of the face bow. Furthermore, the results of this study have the advantage of high

172 applicability in the development of a sophisticated virtual articulator. The final goal of this study was

173 to develop clinical techniques of determining KA of a patient with loss of occlusal support and

174 transferring their KA to an articulator. The concept of the KA was originally found by Kohno [3]

175 from the investigation of sagittal jaw movements in ten subjects with normal dentition and stable

176 occlusal contacts at the ICP, without signs or symptoms of temporomandibular disorders. In this

177 research, all subjects were therefore selected using the same selection criteria for inclusion as those

178 of Kohno's study [3] and the possibility of determining the KAP and the KA from the incomplete

179 jaw movement data without data points in and around the ICP were examined. 
181 vertical deviation of the curve fitting the pathway [14], area enclosed the pathway [15,16], space

volume dictated by the pathway[17], and consistency of the pathways [6,7] have been applied for

fitting the sagittal border movement pathway was constructed, and then the radial thickness of the jaw movement pathway around this circle was evaluated. As shown in Fig. 7, the ref_KAP average of the quadratic mean perpendicular distance between the ref_KAP $\mathrm{m}_{\mathrm{m}}$ and ref_KA of 45 considered valid. a gradual deviation of exc_KAP $\mathrm{m}_{\mathrm{m}}$ from ref_KAP $\mathrm{m}$ in the antero-inferior direction. In a data exclusion area below the $\mathrm{BP}$, the exc_KAP $\mathrm{m}_{\mathrm{m}}$ was located close to the ref_KAP $\mathrm{m}_{\mathrm{m}}$, and was within $1 \mathrm{~mm}$ of 
198 It could be said that if the exclusion area is below the BP, the sagittal border movement can be

199 validly used for an estimation of the KAP. This finding indicates that the incomplete jaw movement

200 data set without the data points in the range of a $7 \mathrm{~mm}$ distance from the ICP can be used for the

201 estimation of the KAP. As a result of this study, the possibility of determining the KA of patients

with loss of occlusal support was suggested.

203

In this research, all subjects had natural dentition and stable occlusal contacts at the ICP.

204 Therefore, it is questionable whether the same results would be obtained with the jaw movement of

205 complete and/or partially edentulous patients who have lost their original ICP. As an earlier study on

206 the jaw movement of completely edentulous patients by the multi-flash method [19] reported that the

207 condylar point movement exhibited a convergence pathway the same as that found in subjects with

patients. Before the clinical application of this technique, further studies to identify the most suitable 
217 This work was supported in part by Japan Science and Technology Agency. (JST), A-step

Conflict of interest

Funding

Ethical approval

Hospital (No.575), Tokushima, Japan

[1] Jarabak JR. An electromyographic analysis of muscular and temporomandibular joint disturbances due to imbalances in occlusion. Angle Orthod 1956;26:170-90. 
234 [2] Wassell RW, Steele JG, Welsh G. Considerations when planning occlusal rehabilitation: a review of the literature. Int Dent J 1998; 48: 571-81.

236 [3] Kohno S. A study on condylar movements with reference to the mandibular movement in sagittal Prosthodont Soc 1968; 12: 350-80.

[4] Kohno S. Analyse der Kondylenbewegung in der Sagittalebene. Dtsch Zahnärztl Z 1972; 27:

[6] Yatabe M, Zwijnenburg A, Megens CC, Naeije M. The kinematic center: a reference for condylar

[5] Kohno S, Bando E, Tanaka B, Kuriyama M, Tabata T. Mandibular movement and mandibular $739-43$.

[7] Pröschel PA, Morneburg T. Investigation of a possible relationship between kinematic points and condylar attachments of the lateral ligaments. J Oral Rehabil 2000; 27: 166-74.

[8] Ishikawa T. Development and application of six-degree-of-freedom jaw tracking device for chair side use with utilizing a pair of triaxial coils. Shikoku Soc Dent Res 2006; 19: 55-66. 
[10] Suzuki A. A study of mandibular movement in six degree-of-freedom with a newly developed jaw movement analyzer. J Jpn Prosthodont Soc 1987; 31: 712-25.

[11] Catic A, Naeije M. Location of the hinge axis and the kinematic centre in asymptomatic and clicking temporomandibular joints. J Oral Rehabil 1999; 26: 661-5.

[12] Lewis RP, Buschang PH, Throckmorton GS. Sex differences in mandibular movements during opening and closing. Am J Orthod Dentofacial Orthop 2001; 120: 294-303 Prosthodont Soc 1973;17: 121-5.

[14] Hayashi T, Kato K, Ishioka K. On a method of searching the kinematic axis point using 1994; 6: 199-208. 

axis of the temporomandibular joint. Front Med Biol Eng 1996; 7: 21-33.

271 [18] Nishi K, Hayashi T, Ishioka K. A method of analysing condylar movement: kinematic axis point as a kinematically analytic point. J Jpn Prosthodont Soc 1998; 32: 1411-19.

273 [19] Suetsugu T. A study of the movement and the jaw relation of edentulous mandible in sagittal plane with the multiple electronic flush apparatus. J Jpn Prosthodont Soc 1961; 5: 131-169. 
Fig. 1. A six degree-of-freedom jaw tracking device using a magnetic sensor system.

a: primary sensor unit $\quad$ b: secondary sensor unit

Fig. 2. Reference coordinate system and intercondylar axis determined based on the Bonwill triangle and the Balkwill angle.

Three orthogonal axes show the upper jaw coordinate system $\left(\mathrm{O}_{\mathrm{u}}-\mathrm{x}_{\mathrm{u}} \mathrm{y}_{\mathrm{u}} \mathrm{z}_{\mathrm{u}}\right)$. The $\mathrm{x}_{\mathrm{u}} \mathrm{z}_{\mathrm{u}}$ and $\mathrm{x}_{\mathrm{u}} \mathrm{y}_{\mathrm{u}}$ planes are the sagittal (SAG) and horizontal (HOR) planes, respectively. The equilateral triangle $\mathrm{IN}-\mathrm{P}_{2}-\mathrm{P}_{12}$ is the Bonwill triangle with a side length of $110 \mathrm{~mm} . \angle \alpha$ is a Balkwill angle of $20^{\circ}$. Line $\mathrm{P}_{2}-\mathrm{P}_{12}$ is the initial intercondylar axis (ICA) and $\mathrm{P}_{\mathrm{m}}(\mathrm{m}=1 \ldots 13)$ is a distinct point arranged at $10-\mathrm{mm}$ intervals on the ICA, and $P_{2}$ and $P_{12}$ are the right and left condylar points, respectively. $V_{m}$ is the sagittal plane that passes through $\mathrm{P}_{\mathrm{m}}$ and is perpendicular to the ICA.

Fig. 3. Radial thickness of the sagittal border movement pathway.

The least squares circle fitting the $2 \mathrm{D}$ projection of sagittal border movement data is found. The circumference is divided into $\mathrm{n}$ arcs of $0.2 \mathrm{~mm}$ using dotted rays that start at the center $(\mathrm{C})$ of the least squares circle. The maximum radial thickness $r_{i}(i=1 \ldots n)$ of the jaw movement data between two adjacent dotted rays is calculated. The sum of the squares of $\mathrm{rh}_{\mathrm{i}}$ is employed as a geometric parameter to search for the KAP. 
Fig. 4. Searching procedures for the KAP.

The searching procedure for the KAP is started from $5 \times 5$ grid points spaced $10 \mathrm{~mm}$ apart, the center of which was set to $\mathrm{P}_{\mathrm{m}}$ that is on the ICA. $\mathrm{MP}_{\mathrm{m}}$ is the point minimizing the value of the geometric parameters, and is chosen from among the 25 points, and then the same grid analysis performed for 25 new points spaced $5 \mathrm{~mm}$ apart, the center of which is $\mathrm{MP}_{\mathrm{m}}$. This searching procedure is repeated nine times (grid interval $=0.39 \mathrm{~mm}$ ), and finally, the last $\mathrm{MP}_{\mathrm{m}}$ is selected as the $\mathrm{KAP}_{\mathrm{m}}$. The two perpendicular arrows show the subject's anterior (A) and superior (S) directions, respectively. The length of the arrow indicates $10 \mathrm{~mm}$ of jaw movement pathway.

Fig. 5. Complete and incomplete (excluded) sagittal border movement of the incisor point in the sagittal view.

A: The complete sagittal border movement. B: The excluded sagittal border movement.

Fig. 6. Deviation of the exc_KAP estimated from the incomplete sagittal border movement from the reference KAP (ref_KAP).

Antero-posterior $(\mathrm{dx})$ and supero-inferior $(\mathrm{dz})$ positional deviations and Euclidean distances $(\mathrm{d})$ between exc_KAP $(\bullet)$ and ref_KAP $(0)$ were calculated. 
Fig. 7. Jaw movement pathways of the points on the ICA and the estimated $\mathrm{KAP}_{\mathrm{m}}$.

The estimated $\mathrm{KAP}_{\mathrm{m}}$ demonstrated a narrow, belt-shaped range during the complete sagittal border movement, and was located mostly on the KA.

Fig. 8. Relationship between the data exclusion range and the antero-posterior (dx) and supero-inferior (dz) positional deviations and Euclidean distance between exc_KAP and ref_KAP. There were two linear relationships in the data with a sudden change in the slope. Data are plotted as the mean \pm SD. 
Fiqure

.

.

(n)

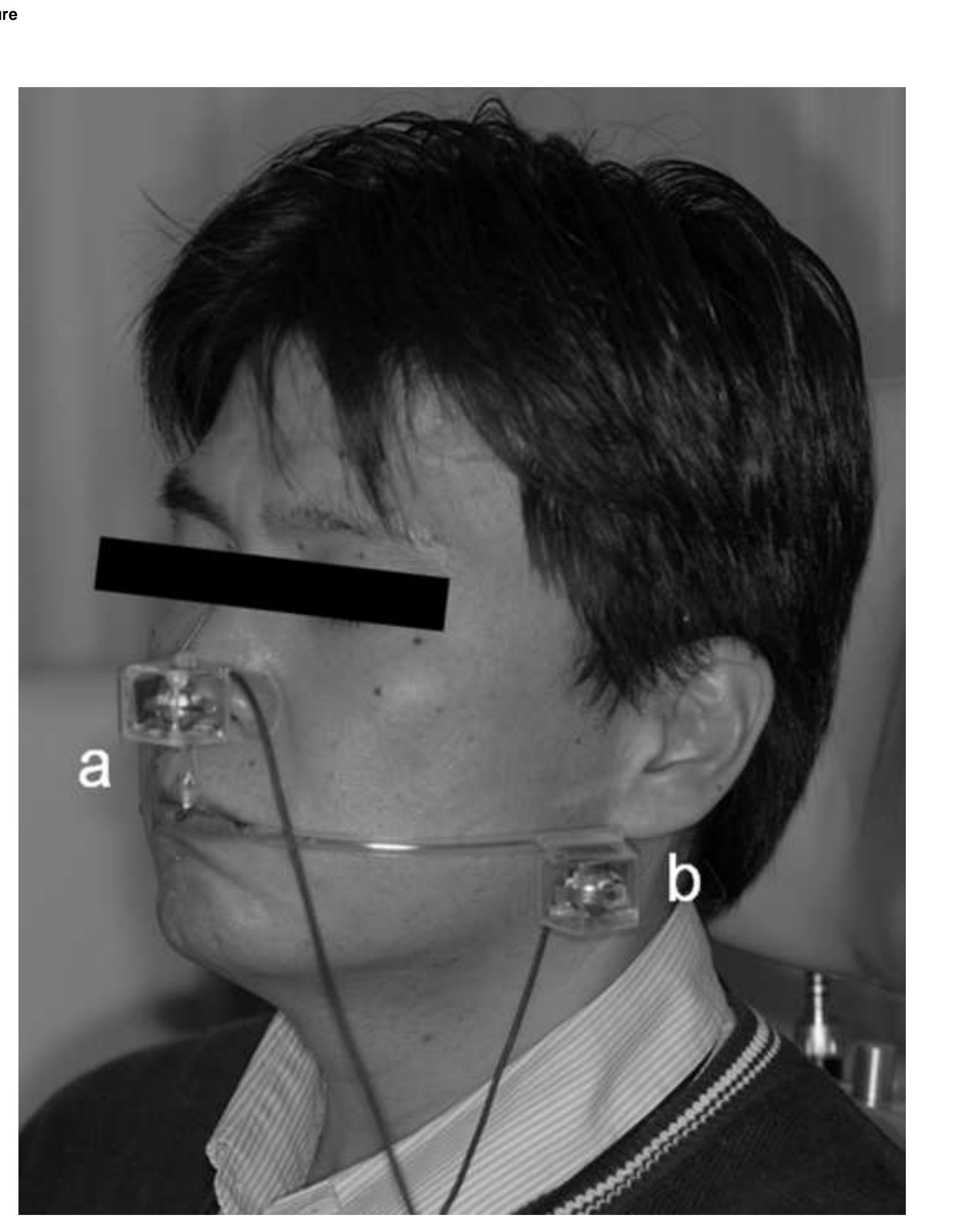

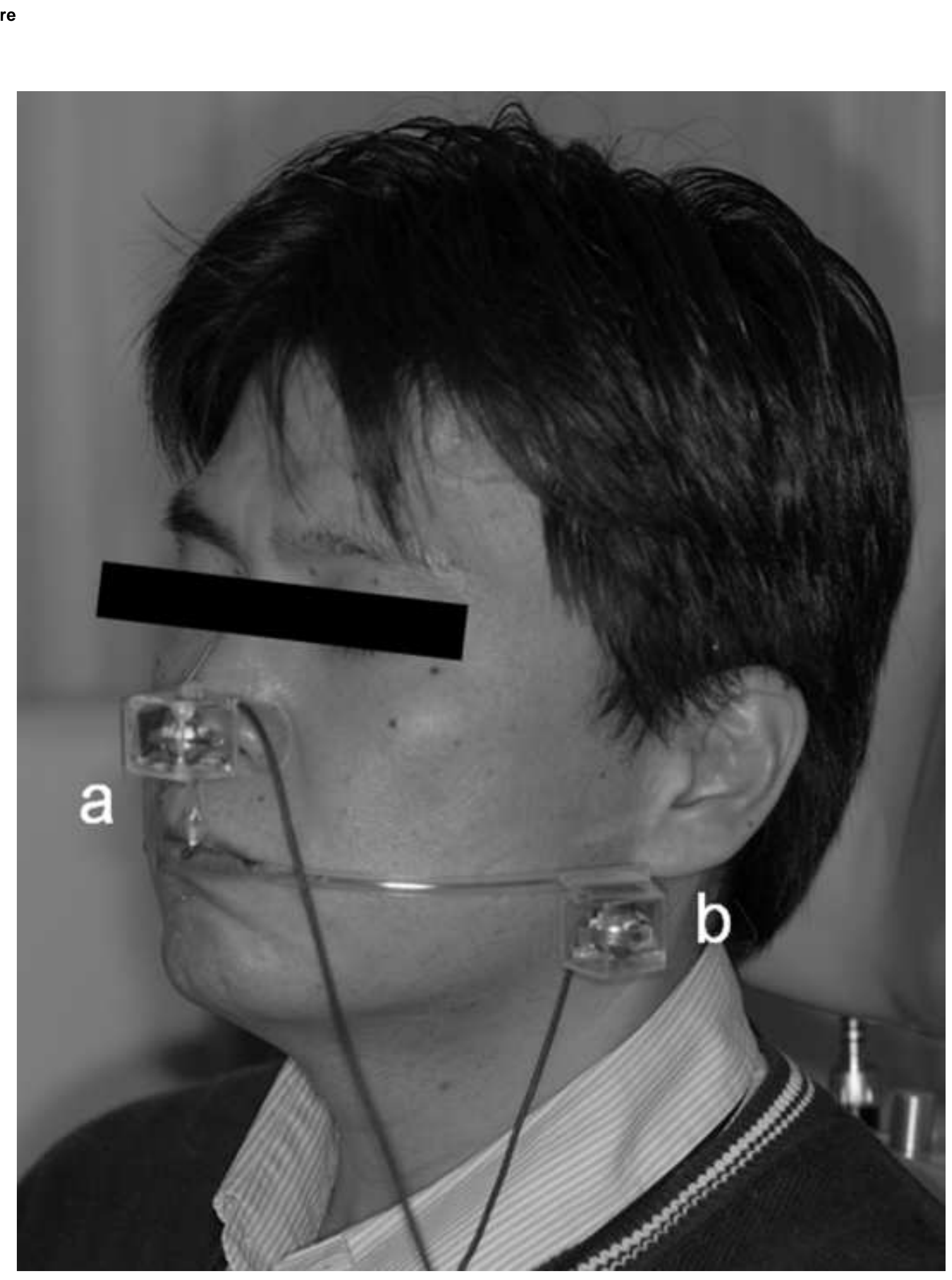

(⿸丆口

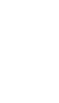

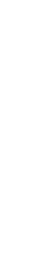


Intercondylar axis (ICA)

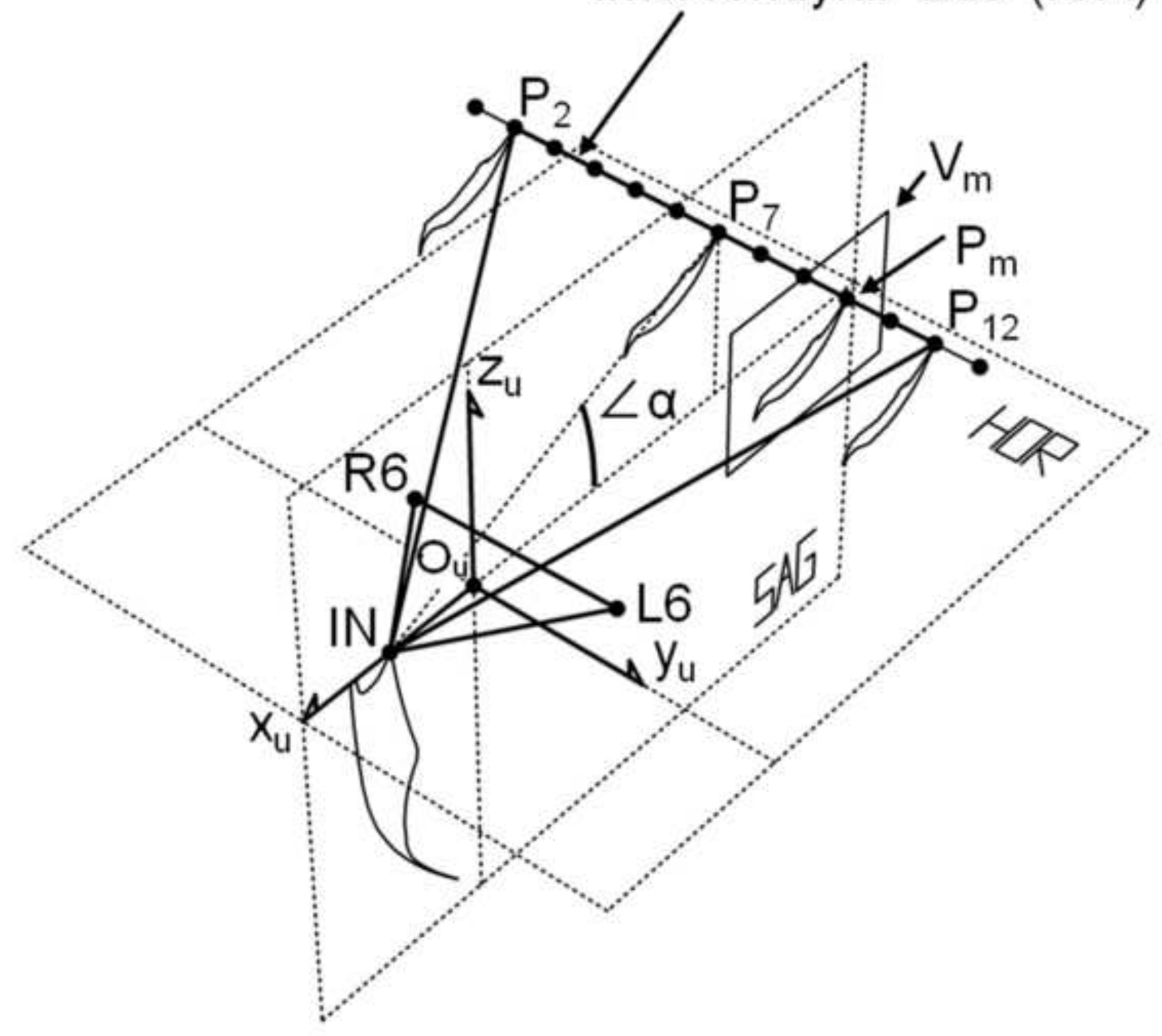




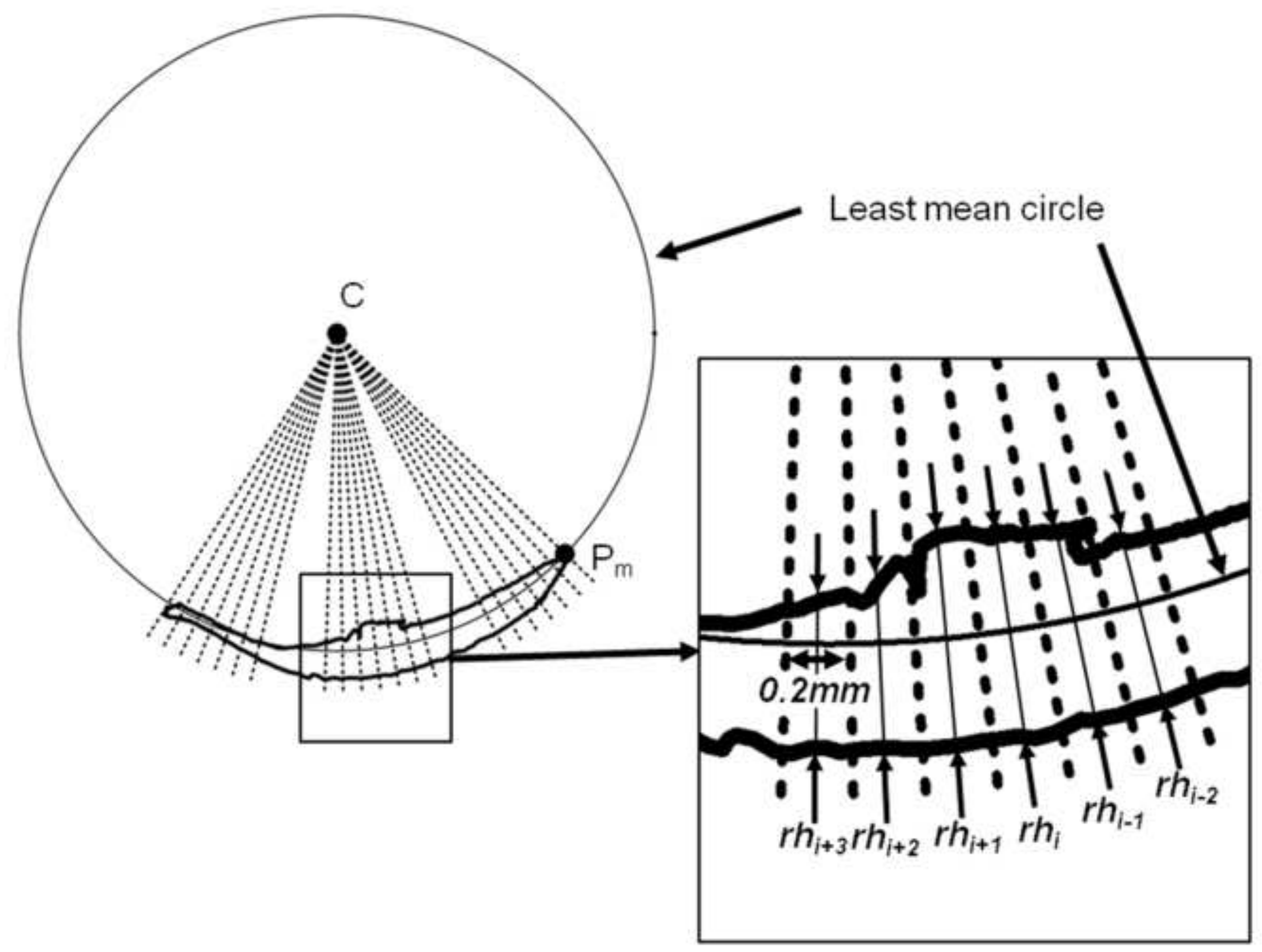




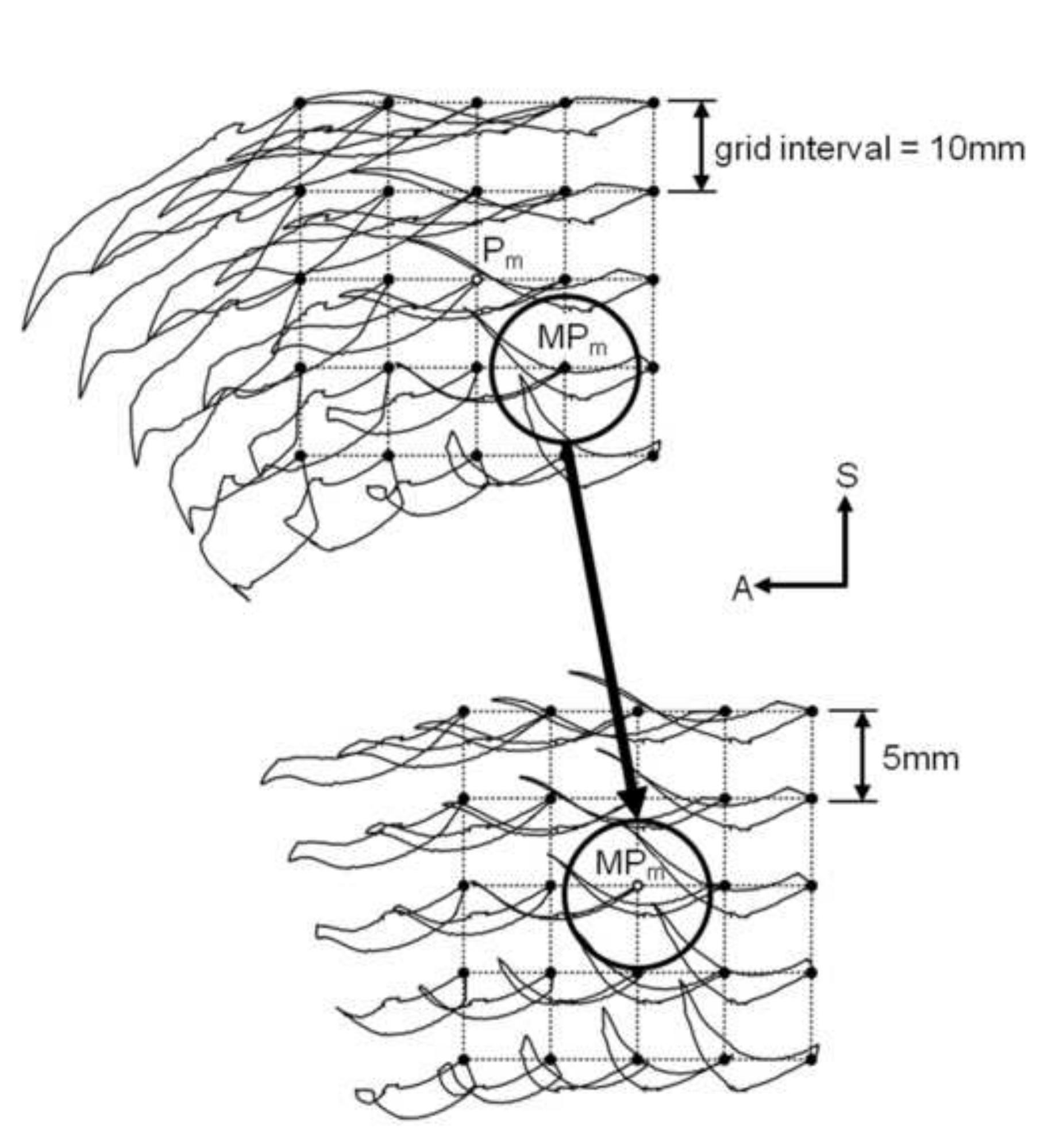

Figure

.

.

\section{Figur}
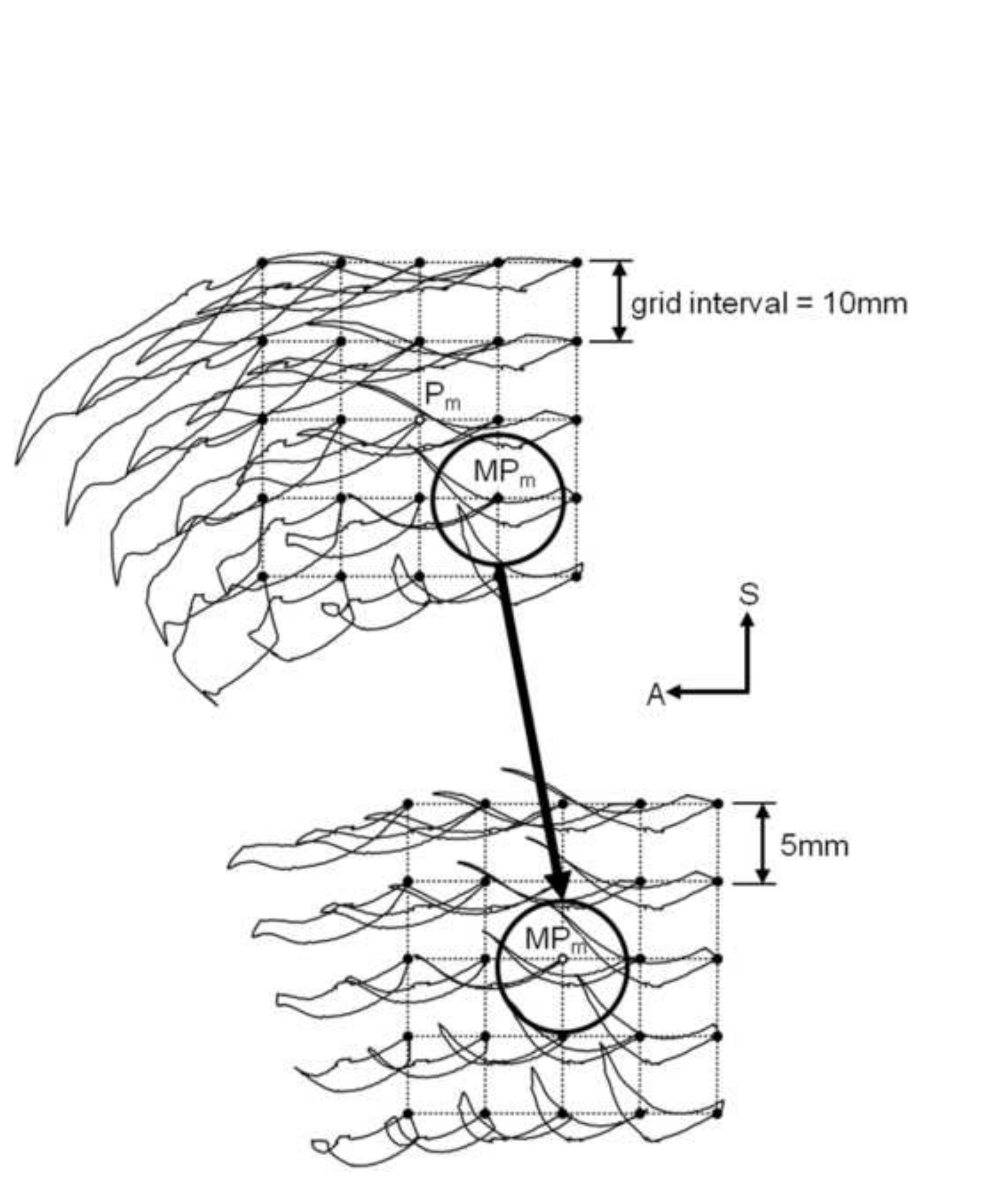


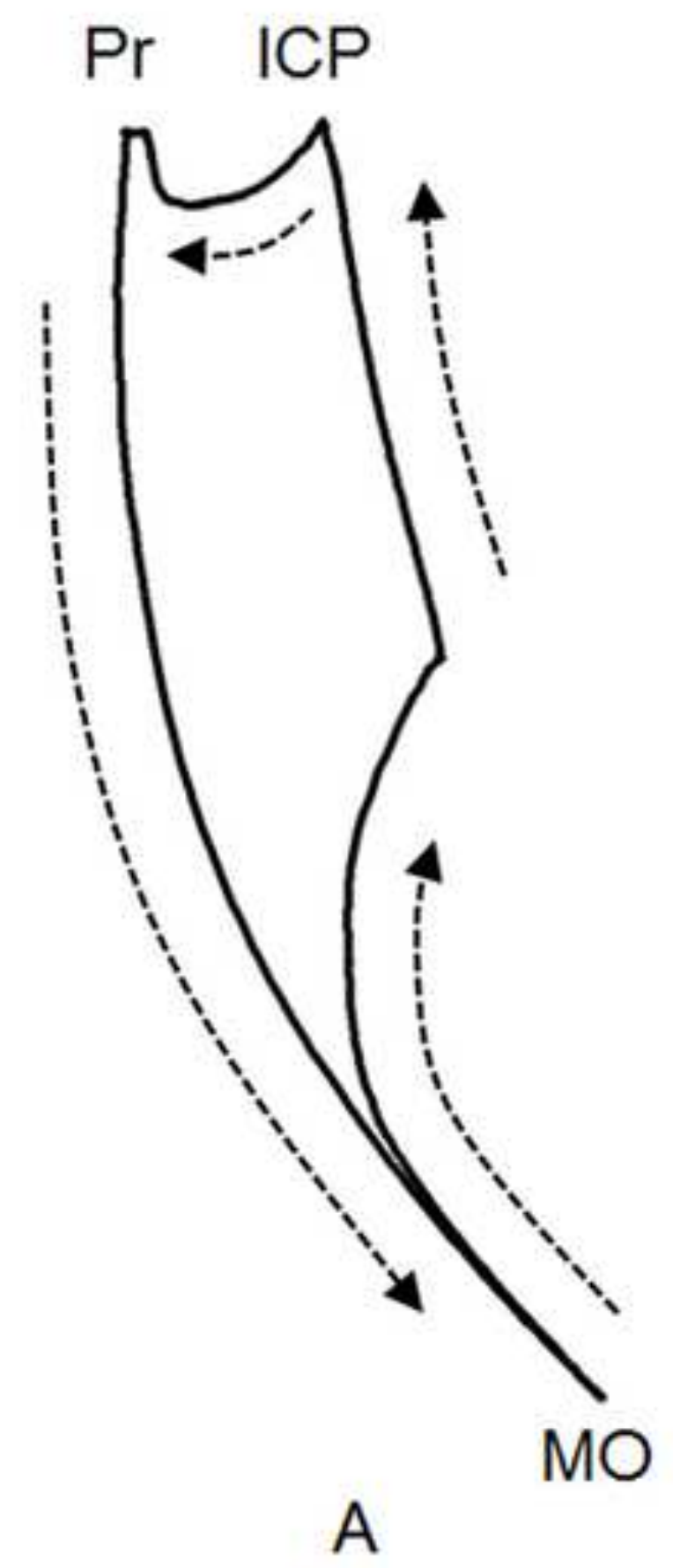

Pr ICP

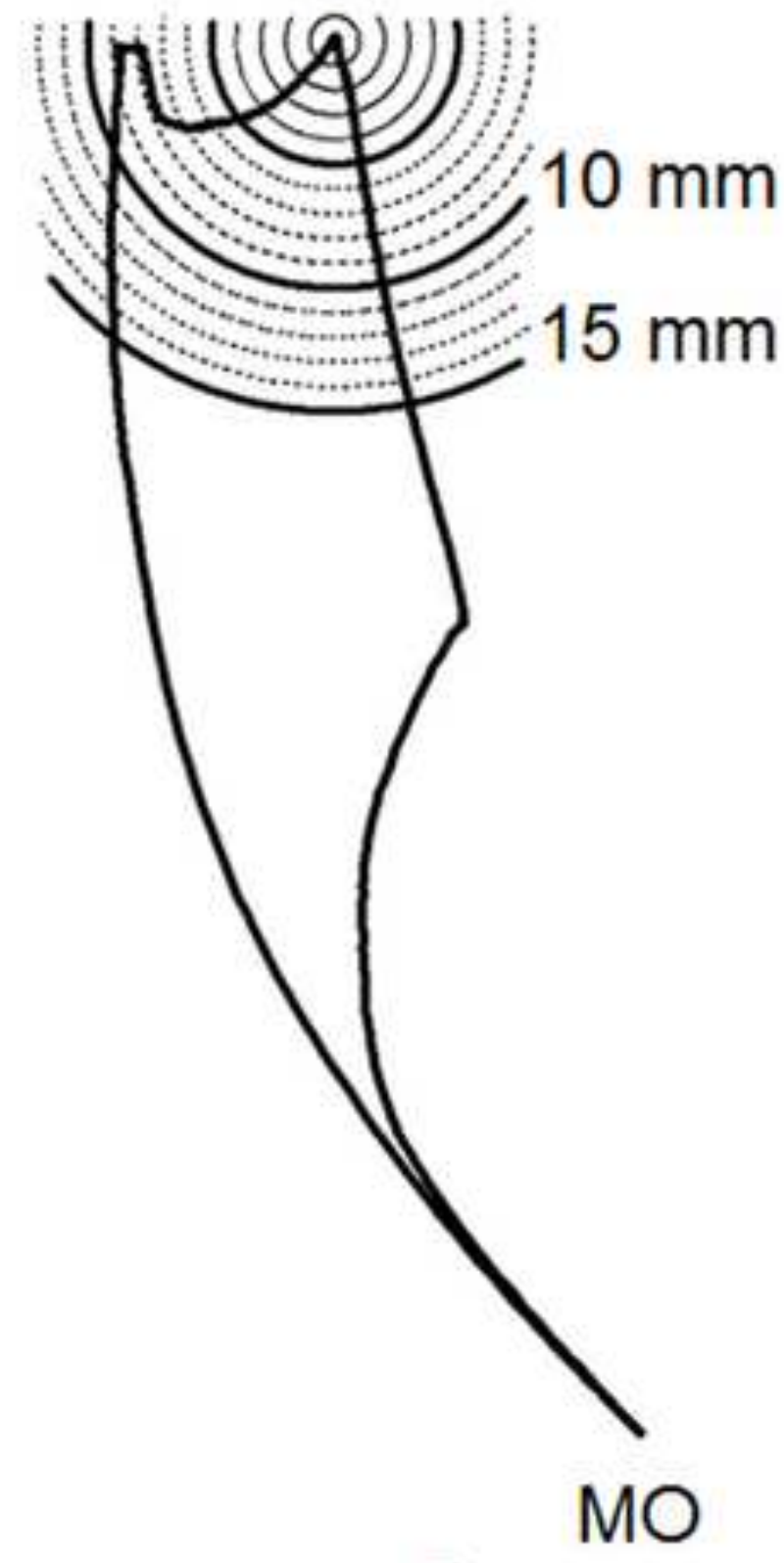

B 


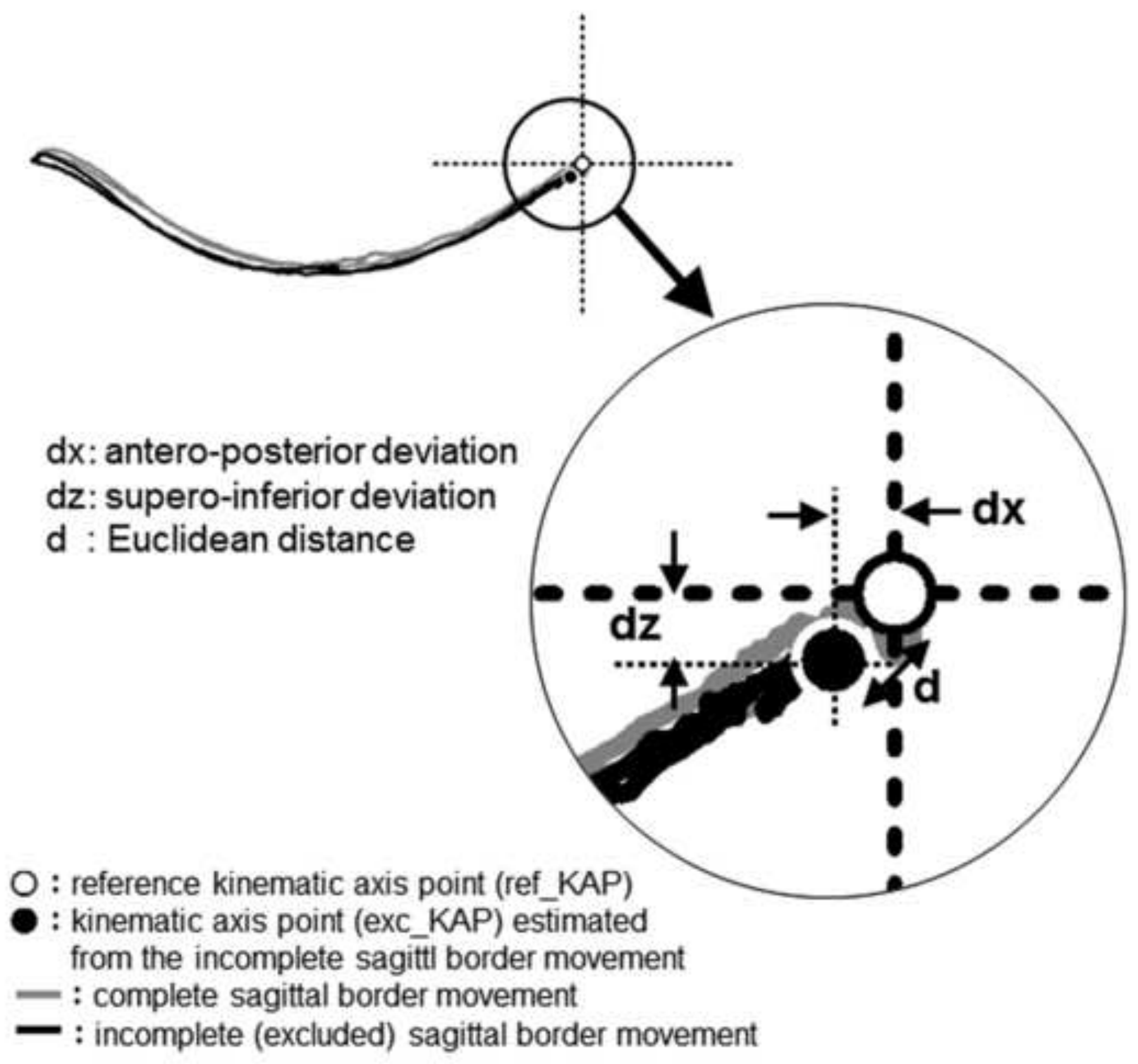




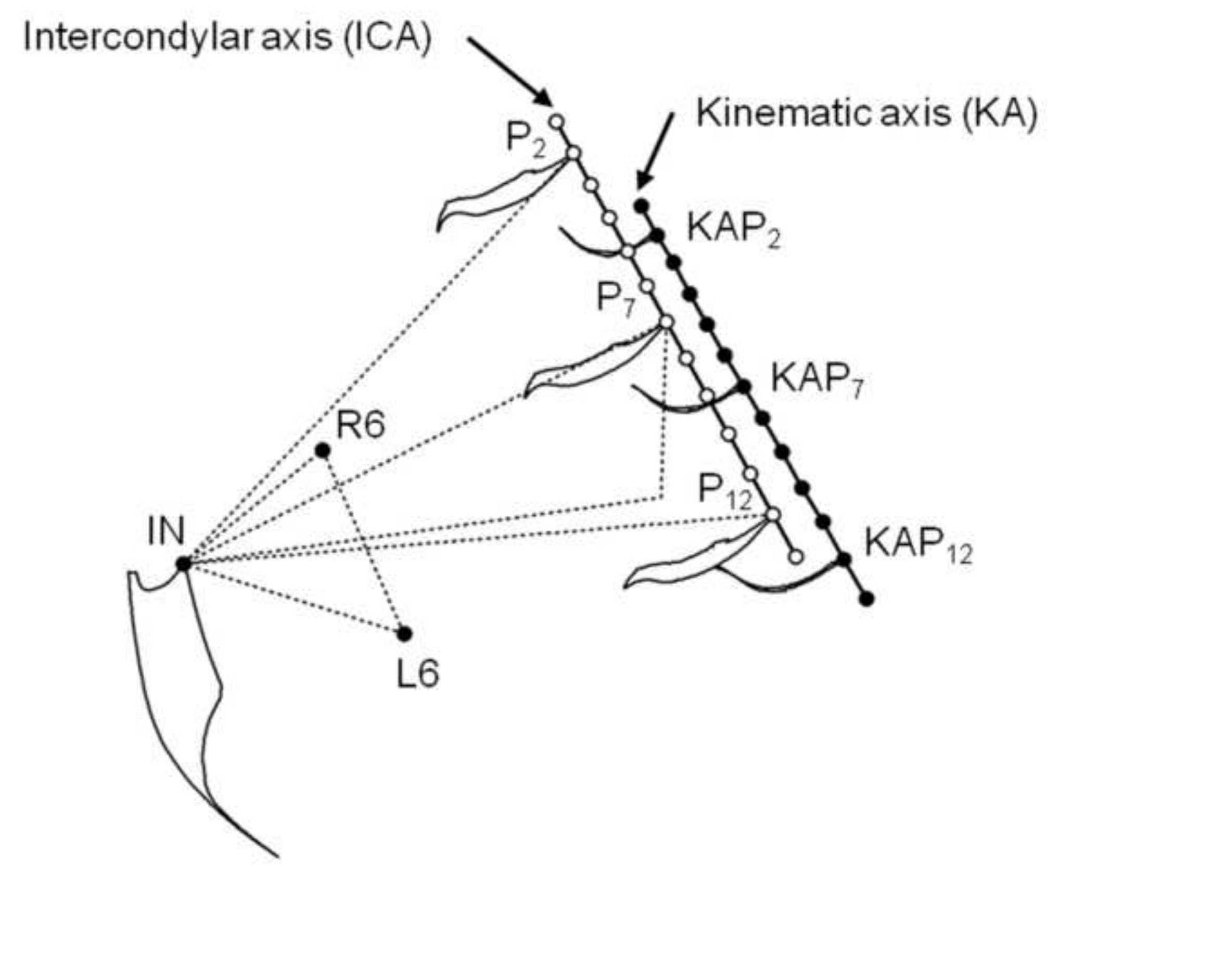

interc

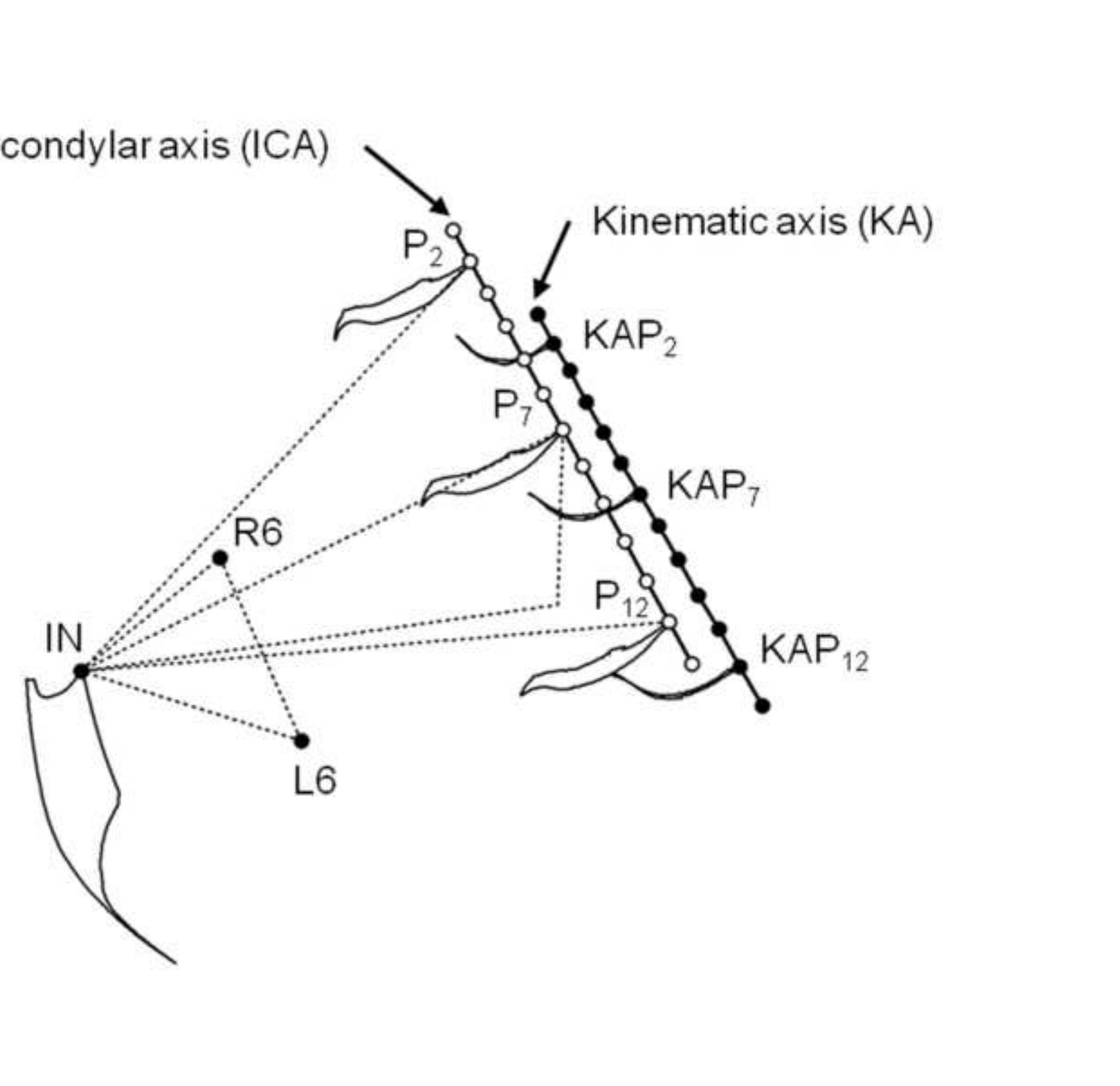

.

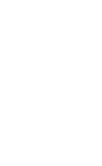

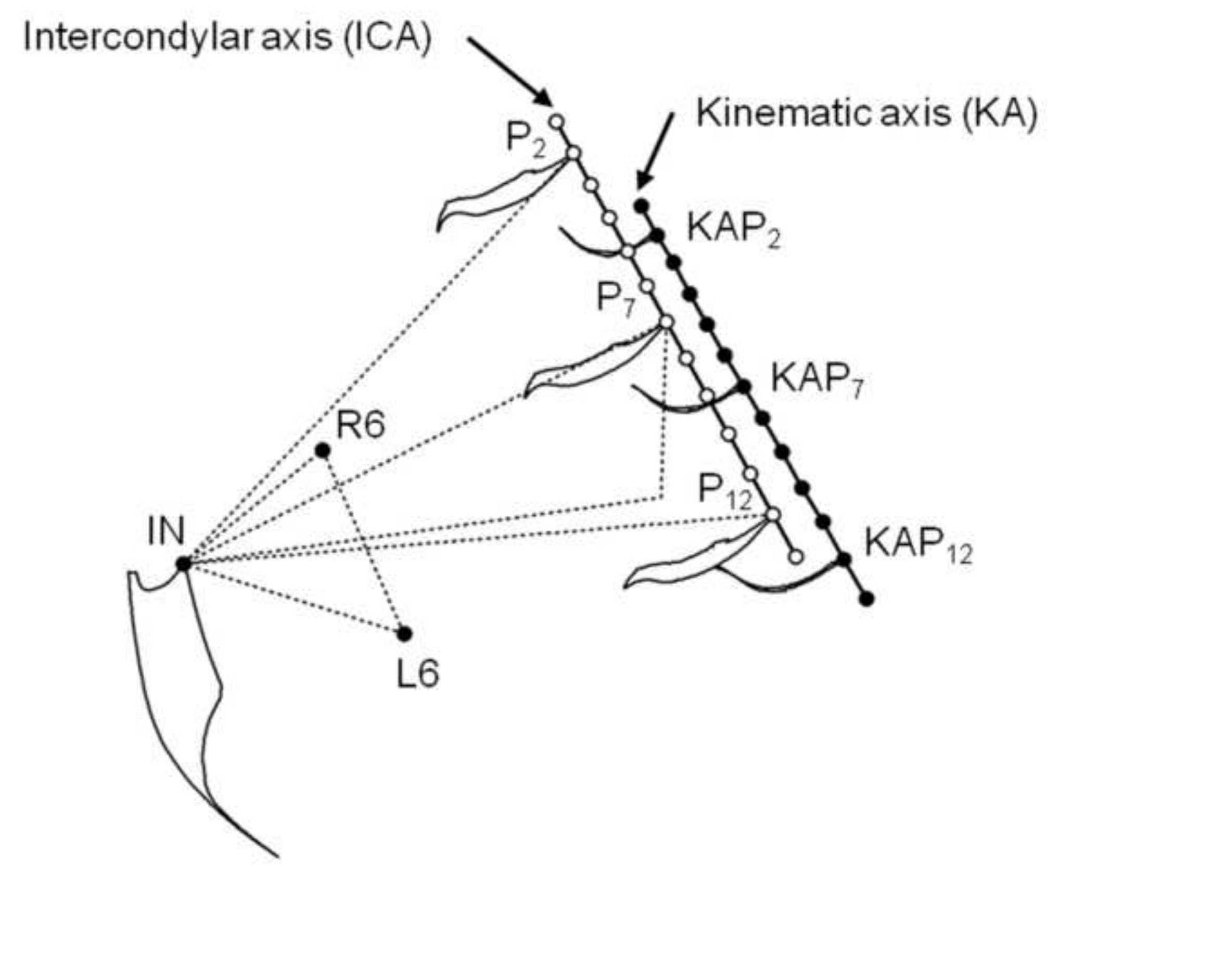



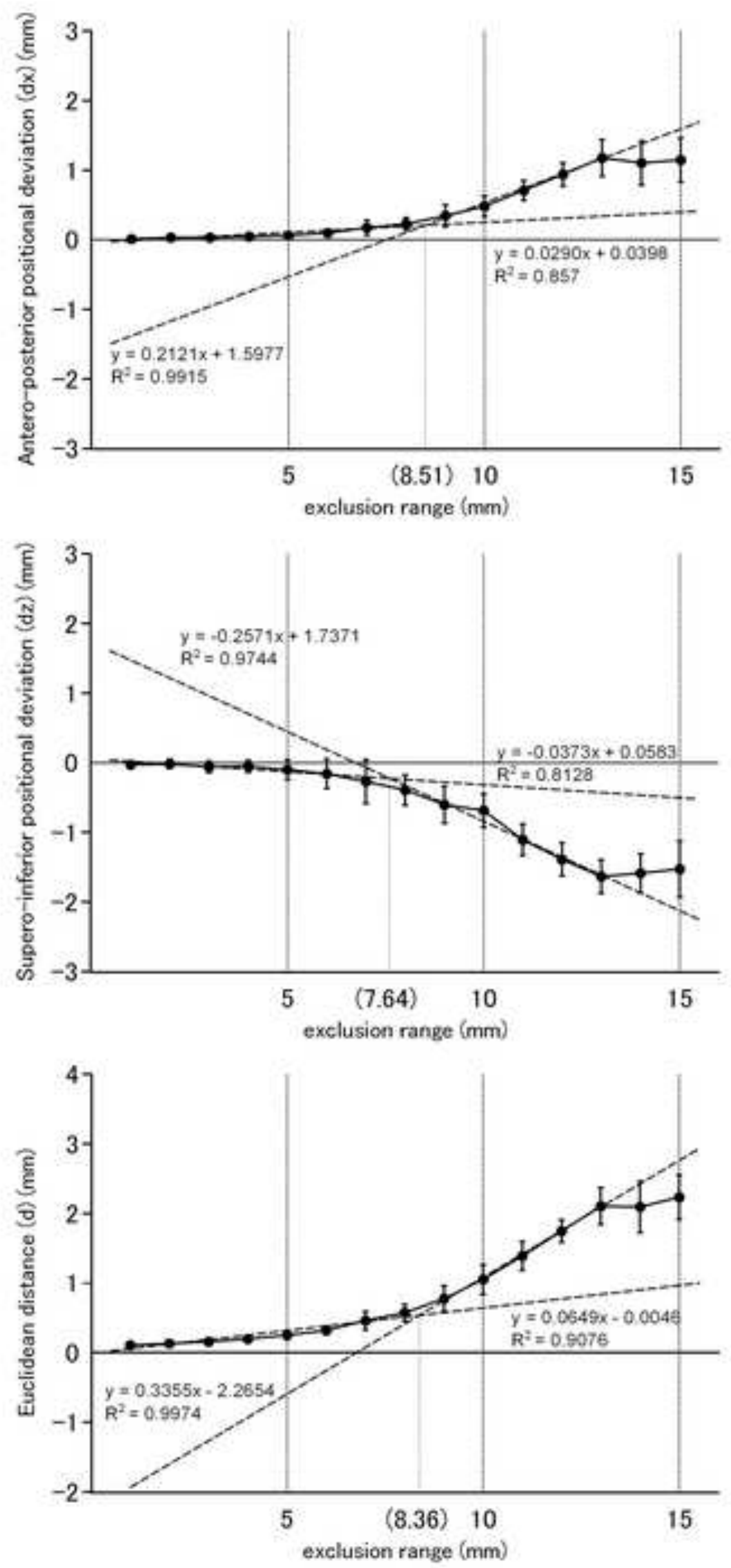